\title{
Impacto de los hábitos lectores en los estudiantes de bachillerato en época del covid-19. 2020
}

Impact of reading habits on high school students in the time of covid-19. 2020

Lucia Guadalupe Macías Mendoza. ${ }^{1}$ \& Viviana Navarro Cedeño. ${ }^{2}$

\begin{abstract}
.
DOI: $\underline{\text { https://doi.org/10.33262/concienciadigital.v4i4.1894 }}$

Introduction. Reading is considered a connection between the person who is reading and the text, producing significant learning and that is where an exploration of ideas is established and new tastes for reading emerge. Objective. Analyze the impact of reading habits on high school students from the Pedro Zambrano Barcia educational unit at the time of covid-19. Methodology. In the development of this research, a methodology that has a quantitative and descriptive approach is applied, using techniques such as the survey and contrasted in the questionnaire that was applied through the google forms platform. Conclution. According to the results, it is considered that the participating students have a medium reading level and that it is developed as a result of the COVID-19 problem, that is, their academic works require them to have close encounters with readings that are key to the development of their academic activities. The pandemic has allowed the education system to migrate its learning system, opening the way to the construction of an academic, formative and intersectional reading as a reading impact.
\end{abstract}

Keywords: Reading Habit, Learning Development, Reading Levels, Covid-19.

\footnotetext{
${ }^{1}$ Universidad Técnica de Manabí, Manabí, https://orcid.org/0000-0003-4625-3946.

2 Universidad Técnica de Manabí, docente de la Facultad de Ciencias Humanísticas y Sociales vivíana.navarro@utm.edu.ec https://orcid.org/0000-0001-8431-2965
} 


\section{Resumen.}

Introducción. La lectura es considerada una conexión entre la persona que está leyendo y el texto, produciendo un aprendizaje significativo y que es ahí es donde se establece una exploración de ideas y surgen nuevos gustos para leer. Objetivo. Analizar el impacto de los hábitos lectores en los estudiantes de bachillerato de la unidad educativa Pedro Zambrano Barcia en época del covid-19. Metodología. En el desarrollo de esta investigación se aplica una metodología que tiene un enfoque cuantitativo y descriptivo, utilizando técnicas como le encuesta y contrastado en el cuestionario de preguntas que fue aplicado mediante la plataforma de google forms. Conclusión. De acuerdo con los resultados se considera que los estudiantes participantes tienen un nivel de lectura medio y que se encuentra desarrollado a raíz de la problemática por COVID-19, es decir sus trabajos académicos les exigen que tenga encuentros cercanos a lecturas que son claves para el desarrollo de sus actividades académicas. La pandemia ha dejado que el sistema de educación migre su sistema de aprendizaje, abriendo paso a la construcción de una lectura académica, formativa e interseccional como impacto lector.

Palabras claves: Habito lector, Desarrollo de aprendizaje, Niveles de lectura, Covid-19.

\section{Introducción.}

La humanidad sufre hoy en día una situación grave como es la del COVID-19, es un virus poco conocido, el SARS-CoV-2, que ha provocado millones de fallecidos, incluidos jóvenes. La Organización Mundial de la Salud (OMS) manifiesta que el Covid-19 no va a desaparecer, es decir, la pandemia puede convertirse en endemia y para ello la sociedad debe estar preparada para convivir con este virus en diferentes países (Velázquez, 2020).

Cuando apareció el COVID-19 notificado en la ciudad de Wuhan China, el 31 de diciembre del 2019 la enseñanza cambio en su totalidad, lo que afuera de las aulas a veces se vive como un aplanamiento de la oralidad por un uso no ecológico de la red, se debe convertir dentro de la enseñanza virtual en un favorecimiento de los rasgos humanísticos, entendidos como la capacidad del maestro para motivar al estudiante y favorecer su interés y curiosidad por el descubrimiento de nuevos saberes (Torres, 2020).

Guardiola \& Baños (2021) manifiesta que la lectura durante la pandemia de COVID-19 ha logrado despertar el interés por estudios que se encuentren realizados por plataformas de lectura digital han señalado un incremento lector en este soporte, que ha llegado a duplicarse en estos tiempos. El confinamiento causado por la emergencia sanitaria, ha cambiado las formas de leer, siendo estos parten de una migración en el formato digital; mucha gente ha optado leer en bibliografía en formato electrónico más reconocida como lectura digital y lo ha tomado como un hobbie.

El grupo de investigación: Estructura de Recerca Interdisciplinar (ERI)- Lectura (2020) menciona que "la evolución de las diferentes tipologías de lectura siguió patrones 
diferentes a medida que avanzó el confinamiento. La lectura de ocio aumentó de manera generalizada las dos primeras semanas de confinamiento" (p.26).

La lectura se ha convertido en algo esencial en estos tiempos, ya sea para estar informado por noticias, para el trabajo, para las instituciones educativas o simplemente para tener una forma de distraerse sanamente durante el confinamiento.

La lectura también puede definirse como la acción recurrente de recibir y asimilar información de terceros mediante la escritura, lo que quiere decir que contiene dos componentes: la rutina y el entendimiento que se refiere a comprender y reflexiona sobre lo leído (Venegas, 2019).

Guerrero, Valledor \& Ponce de León (2018), mencionan que el hábito desarrolla en la persona la metacognición de acuerdo a lo que realiza cotidianamente. En el desarrollo de éste, se deben considerar principios como estimular a los educandos a que lean sistemáticamente, ejercitar la lectura para lograr la automatización de las operaciones del proceso lector, conocimientos previos del lector, entre otros.

Osorio (2019) expone algo similar al sostener que el hábito lector, va más allá del propio aprendizaje técnico de la lectura, debido a que supone el paso de una leer esencialmente pasivo a hacerlo vivo y activo, que profundiza en el texto, intercambia experiencias e inquietudes, lo que posibilita comprender y analizarlo desde una visión particular y contextualizada.

La correlación que existe en el hábito lector, es sin duda de carácter formativa, pero sin embargo el autor Paredes (2015), expresa que el acto de leer en un proceso significativo, donde tiende a desarrollar un espacio de lectura, es decir en la vida cotidiana, posee un proceso de aprendizaje y luego su fijación, para convertirse en una necesidad. La persona que lee, se ve impulsado a investigar, es decir, siente necesario conocer algo.

Se estima que una persona tenga neto gusto por la lectura, más no porque tenga que hacerlo. Así refieren Garzón \& López (2019) el hábito de leer, es hacerlo voluntariamente mas no por obligación, realizándolo como un ejercicio libre donde el lector disfruta, se enriquece en cuanto a su vocabulario y adquiere nuevos conocimientos. Al mencionar esto, significa que lo realiza constantemente y forma parte de sus actividades en donde su principal objetivo es interpretar la información receptada, lo cual despierta y mejora las habilidades cognitivas que conforman su enseñanza y aprendizaje.

Así mismo, Vélez (2018) expresa que "algo similar al apartado anterior: el hábito lector mejora la velocidad lectora, utiliza técnicas, reconoce estructuras, se vale de recursos para la comprensión; los cuales preparan a la producción oral o escrita" (p.5). La lectura no puede ser un acto impuesto, sino la introducción al conocimiento de un mundo de agrado para cada estudiante. Por tal razón, todo educando debe discernir sobre aquellos libros que le despierten su interés, dentro de la variedad de textos con el respeto de sus gustos y competencias lectoras. 
Como se manifiesta, el interés por leer es el factor más importante para crear hábitos de lectura. Surge de relacionar este acto con la satisfacción, a la sensación de logro y al entretenimiento. La forma de aprender y de crear un hábito en el individuo, forman parte de diversos y amplios procesos de intercambio de ideas entre sujetos y de los mismos con ciertas instituciones y escenarios; donde circulan los objetos, los productos, mediadores y facilitadores, relacionados con la práctica lectora. (Hilario, 2016)

Construir hábitos de lectura integra un apoyo indispensable en el desarrollo de habilidades intelectuales y estratégicas que les permitan a las personas participar de manera activa en el progreso de la sociedad, puesto que la lectura es un instrumento primordial para el crecimiento personal y social de los individuos. (Guerrero \& Ponce de León, 2018)

Del mismo modo Camacho (2016) analiza que el hábito lector debe su origen a la relación que una persona llega a establecer con un libro, puesto que al convertirlo en parte de su cotidianeidad; se convierte en partícipe y orientador de sus actividades. Además, es propicio el estímulo que los padres y docentes deben hacer para llegar a determinar este comportamiento, no solo por méritos académicos, sino más bien por beneficio y aprendizaje propio.

Leer es una habilidad que apasiona desde que el niño está en el vientre de su madre hasta la vejez, de ahí su valor en el mundo. Así lo menciona Alvarado (2016): es un medio por el cual todas las personas aprenden, imaginan, a su vez pueden comunicar, comprender y expresar lo que sienten, entonces es muy importante saber que no es solo una acción ejecutada cuando se estudia, sino también es una forma de divertirse ya sea esta un cuento, una historieta, un poema o una novela.

Este proceso, ayuda mucho al desarrollo cognitivo tanto en los niños como en los adultos, su mente se enriquece, se amplia, gracias a las nuevas experiencias que le transmite al lector. La lectura da apertura al aprendizaje, imaginación, innovación y creatividad, características de un pensador crítico, así como requerimientos de un mundo globalizado para ser deseable, y permanecer competitivo.

Es importante considerar la lectura esencial en el desarrollo y formación para los jóvenes para poder enfrentar a los desafíos en la era del conocimiento, en los niveles académicos es necesario para el óptimo aprovechamiento de los alumnos, de la misma manera para el mejoramiento de su desempeño intelectual y cognitivo. (Flores, 2016).

Del mismo modo, Del Valle (2016) refiere que leer es el encuentro del lector con el texto, entendiendo así que es un proceso complejo en el que intervienen factores internos y externos; se puede considerar individual y también colectivo, ya que la persona interactúa con lo que se lee mediante el conocimiento previo y el contexto, el cual contribuye a que quien está leyendo, construya el significado a través de la relación con su experiencia, con sus conocimientos y sus competencias lingüísticas.

En este sentido, Durango (2015) postula tres niveles de lectura: literal, inferencial y crítica: 
- La lectura literal es el nivel básico centrado en las ideas y la información que se expone en el texto.

- La lectura inferencial constituye lo implícito del texto y requiere un alto grado de abstracción por parte del lector.

- La lectura crítica es de carácter evaluativo donde intervienen los saberes previos del lector, su criterio y el conocimiento de lo leído.

De esta forma, se considera la lectura una actividad formativa, que implica una relación entre lo que está escrito y la subjetividad de quien lee. Esta actividad permite al individuo, tener una gran imaginación, para enriquecer el universo con sus posibilidades recreativas y potenciar la capacidad de crear ideas nuevas, integradas a la experiencia personal. (Jiménez, Fierro, \& García, 2016).

En este sentido, Caride (2015) indica que es necesario que el mundo sea leído e interpretado, con tres propósitos: aportar a un mejor conocimiento de lo cotidiano, de manera diacrónica y sincrónica; activar etapas de cambio que se enfatice en valores e iniciativas con el desarrollo humano y el respeto a la diversidad.

La lectura es una fuente de reducción de deformaciones intelectuales y culturales e indiferencias, y ayuda en la construcción de oportunidades para una vida mejor. En otras palabras, se la reconoce como un recurso importante como los demás, que pueden ser físicos, económicos, científicos y humanos; lo que aporta al progreso de un sujeto. (De Souza, 2016)

Por su parte, Barba Chamorro Morillo Fuertes, \& Realpe (2016) mencionan que como habilidad comunicativa leer influye de manera directa en el proceso de desarrollo de la personalidad de los estudiantes ya que además de modificar las particularidades de su pensamiento, imaginación, memoria y habilidades, también incide en sus sentimientos en sus emociones sus valores y comportamientos. Cuando se enseña a leer, se debe realizar partir de experiencias creativas, que conlleven a la creación de hábitos lectores adecuados en los alumnos.

El ser humano busca estar informado constantemente sobre el mundo y los avances tecnológicos, y para que esto tenga éxito, hay que fomentar la lectura, para dar seguridad, permitir estar preparado y tener conocimiento acerca de lo que acontece en su localidad o fuera de ella. (Figueroa \& Zambrano, 2015)

La pandemia, causada por coronavirus (COVID-19) ha provocado una crisis sin precedentes en la sociedad. En el área de educación, las medidas que los países han adoptado ante la situación, se vincula con suspender las clases presenciales en todos los aspectos, utilizando una variedad de formatos y plataformas virtuales; el apoyo y la movilización del personal y las comunidades educativas, y la atención a la salud y el bienestar integral de las y los estudiantes (CEPAL, 2020)

Hay varias maneras de cambiar una propuesta hacia un modelo asincrónico o sincrónico. Esto depende de la evaluación del tipo de alfabetización digital de los docentes, respecto 
a sus habilidades técnicas y la capacidad que tengan para resolver los inconvenientes que puedan surgir en este proceso (Gagliardi, 2020)

El aprendizaje sufrió una interrupción que difícilmente se puede enmendar con la enseñanza virtual, de ello se han derivado consecuencias significativas para los educandos del último año de Bachillerato que impidieron lograr un desenlace exitoso en su vida académica. Las instituciones educativas deberán evaluar este proceso y tomar los correctivos pertinentes.

Para que los estudiantes puedan acceder a este tipo de educación es necesario contar con las herramientas necesarias que permitan formar parte de las clases virtuales con normalidad, que sea de fácil acceso para las personas que se encuentran enfrentando situaciones difíciles debido a la repentina crisis (Aguirre, Zhindon, \& Pomaquero, 2020)

La constitución del Ecuador en su artículo 26 indica que:

"Suspenderla implica la interrupción del sentido de normalidad y regularidad que favorece el desarrollo y la estabilidad socioemocional de la población en tiempos de crisis"

"Es importante que los maestros interactúen constantemente con los estudiantes y sus familias, con el fin de favorecer la cohesión social, prevenir la violencia intrafamiliar y asegurar la continuidad del aprendizaje". (Unicef, 2020).

El presente trabajo permitió analizar los hábitos lectores en estos tiempos de pandemia, la cual, determinó la relevancia que ha tomado la lectura, puesto que a raíz del confinamiento obligatorio y por ende las suspensiones temporales de las bibliotecas entre otros lugares, dio paso a poder obtener algunos libros por medio de la internet. Esta factibilidad de obtener libros despertó en los adolescentes nuevas formas de distraerse, creando así el hábito de leer. Adicional a ello, cuando empezaron las clases virtuales, ellos pudieron estar atentos a lo que explicaba el profesor y todo ello producto del interés a la lectura. Este criterio se pudo desarrollar a través de la encuesta aplicada a estos adolescentes.

\section{Metodologia.}

Este trabajo fue una investigación de campo que se desarrolló a través de un enfoque cuantitativo y descriptivo, aplico la técnica de la encuesta en la que se utilizó herramientas tecnológicas como es la plataforma de Google forms, con un cuestionario de 15 preguntas realizadas a una población de 122 estudiantes de bachillerato, en donde permitió medir el impacto de los hábitos lectores, las cuales se presentaron estadísticamente y adicional se pudo determinar la situación actual de los estudiantes que fueron parte del objeto de estudio. Este proceso que se desarrolló permitió destacar los resultados que son analizados y procesados en tablas y graficas estadísticas. 


\section{Resultados.}

Tabla 1. Interés de lectura por emergencia sanitaria (Covid-19).

\begin{tabular}{lccc}
\hline \multicolumn{1}{c}{ ITEMS } & CANTIDAD & $\%$ & TOTAL \\
\hline LEER & 28 & $23 \%$ & 28 \\
\hline COMPARTIR CON LA FAMILIA & 58 & $48 \%$ & 58 \\
\hline REDES SOCIALES & 10 & $8 \%$ & 10 \\
\hline APRENDER A COCINAR & 9 & $7 \%$ & 9 \\
\hline DISFRUTAR TIEMPO LIBRE & 17 & $14 \%$ & 17 \\
\hline
\end{tabular}

Elaborado por: Autoras de la investigación (2021).

Fuente: estudiantes de tercero de bachillerato del Colegio "Pedro Zambrano Barcia"

Gráfico 1. Interés de lectura

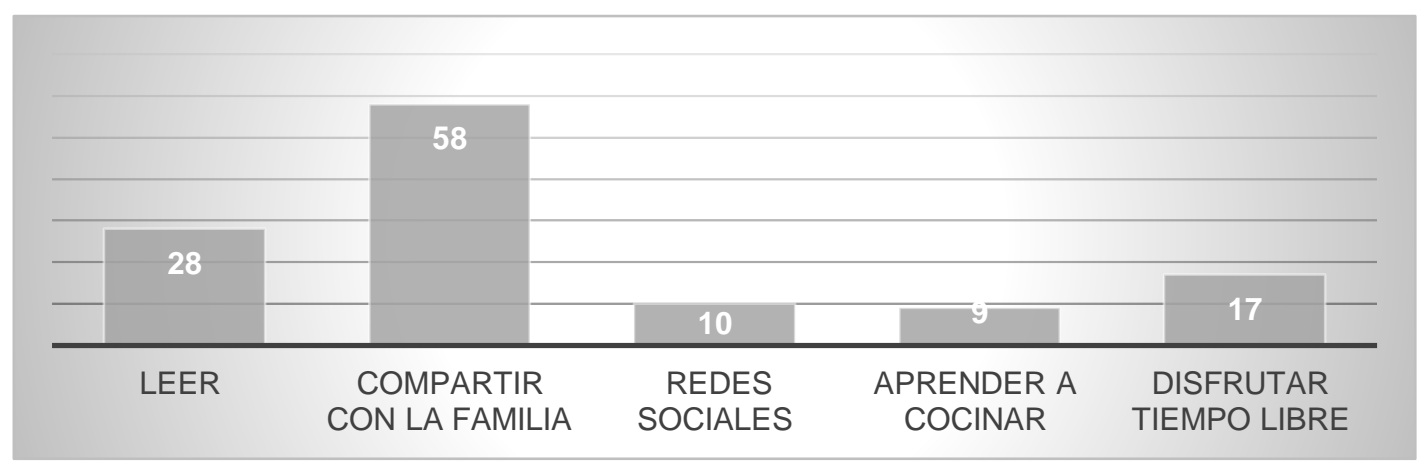

Elaborado por: Autoras de la investigación (2021).

Fuente: estudiantes de tercero de bachillerato del Colegio "Pedro Zambrano Barcia"

\section{Análisis e interpretación de resultados:}

Como se puede observar en la tabla 1 , el $48 \%$ de los estudiantes indicaron que esta época de Covid 19 lo dedicó a la familia, el 23\% afirmó que leyó, es decir que en la lectura encontraron un modo de terapia para poder atenuar la situación de estrés provocada por la emergencia sanitaria, mientras que el $14 \%$ solo disfrutó de sus ratos libres. Estos resultados permiten determinar el tiempo de pandemia en que los alumnos lo dedicaron a varias actividades que fueron relevantes para tolerar el confinamiento, ahora lo que menciona Blum (comunicación personal, 2020), establece que "como familia somos un equipo, entonces el uno recoge los platos, el otro los lava, alguien más hace la comida, y uno más la sirve; en cuanto podamos enseñarnos que somos el primer núcleo de trabajo y que podemos trabajarlo juntos, de esta crisis vamos a salir fortalecidos".

Tabla 2. Percepción de la lectura en la emergencia sanitaria (covid-19)

\begin{tabular}{lccc}
\hline \multicolumn{1}{c}{ ÍTEMS } & CANTIDAD & $\%$ & TOTAL \\
\hline ESTADO DE ÁNIMO & 16 & $13 \%$ & 16 \\
\hline DISTRACTOR & 25 & $20 \%$ & 25 \\
\hline MEJOR COMPRENSIÓN LECTORA & 66 & $54 \%$ & 66 \\
\hline SIMPLE DISTRACTOR & 10 & $8 \%$ & 10 \\
\hline
\end{tabular}


Tabla 2. Percepción de la lectura en la emergencia sanitaria (covid-19) (continuación)

\begin{tabular}{lccc}
\hline ÍTEMS & CANTIDAD & $\%$ & TOTAL \\
\hline DISTRACTOR ABURRIDO & 5 & $4 \%$ & 5 \\
\hline
\end{tabular}

Elaborado por: Autoras de la investigación (2021).

Fuente: estudiantes de tercero de bachillerato del Colegio "Pedro Zambrano Barcia"

Gráfico 2. Percepción de la lectura.

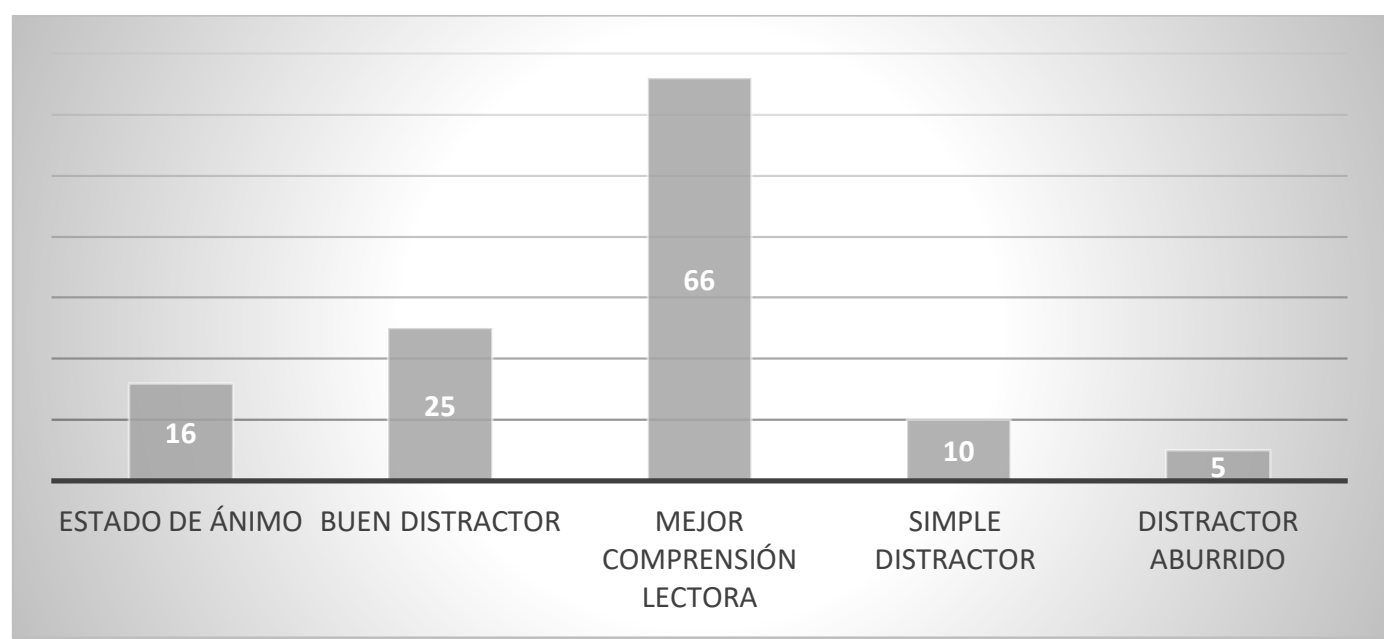

Elaborado por: Autoras de la investigación (2021).

Fuente: estudiantes de tercero de bachillerato del Colegio "Pedro Zambrano Barcia"

\section{Análisis e interpretación de resultados:}

Se aprecia, que el 54\% de los estudiantes expresaron que la lectura les permitió mejorar la comprensión lectora, el $20 \%$ indicaron que es buen distractor y el $13 \%$ contestó que mejora su estado de ánimo. En este sentido, Venegas (2019) menciona acerca de comprender lo leído: también puede definirse como la acción recurrente de recibir y asimilar información de terceros mediante la escritura, lo que quiere decir que contiene dos componentes: la rutina y el entendimiento que se refiere a reflexionar sobre lo que se leyó.

Tabla 3. Tipos de libros leídos en la emergencia sanitaria (covid-19)

\begin{tabular}{|c|c|c|c|}
\hline ÍTEMS & CANTIDAD & $\%$ & TOTAL \\
\hline DIGITALES & 90 & $74 \%$ & 90 \\
\hline FÍsICOS & 32 & $260 \%$ & 32 \\
\hline
\end{tabular}

Elaborado por: Autoras de la investigación (2021).

Fuente: estudiantes de tercero de bachillerato del Colegio "Pedro Zambrano Barcia" 


\section{Gráfico 3. Tipos de libros}

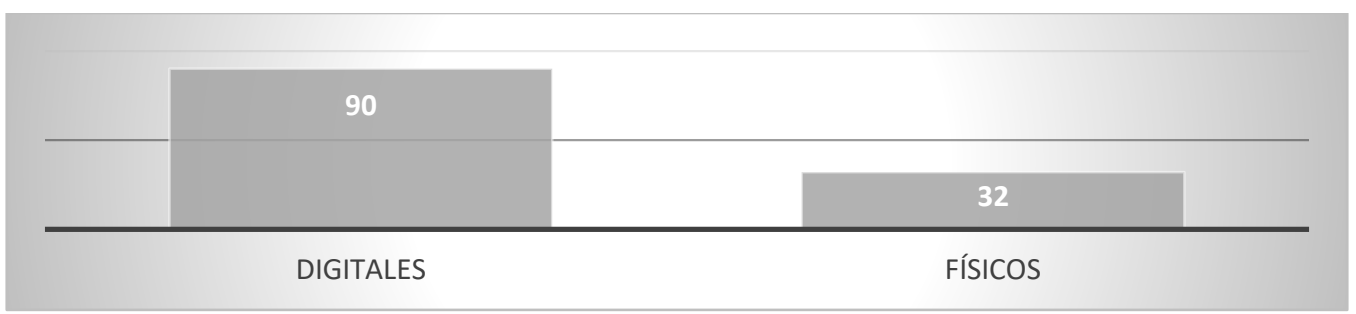

Elaborado por: Autoras de la investigación (2021).

Fuente: estudiantes de tercero de bachillerato del Colegio "Pedro Zambrano Barcia"

\section{Análisis e interpretación}

Se puede afirmar que el $74 \%$ de los estudiantes contestaron que han optado por leer libros digitales, puesto que, por efecto de la emergencia sanitaria actual, las bibliotecas y demás lugares han cerrado temporalmente y aquello ha causado interesarse por este tipo de formatos, mientras que el $26 \%$ expresó que leen textos en físico. Ante esta realidad, Alonso \& Federico (2020), sostienen que los contenidos multimedia educativos, culturales y de entretenimiento a los que pueden tener acceso el público infanto-juvenil se distribuyen en múltiples formas, existe gran variedad de alternativas en video, aplicaciones y podcast-radio, todos ellos disponibles desde celulares y otros dispositivos. Esto ayuda a crear dinamismo en los entornos de educación formales e informales, además fomentan la interconectividad y permiten innovar o mantener, incluso en tiempos de incertidumbre, comunidades culturales y de aprendizaje interconectadas.

Tabla 4. Lectura digital y el conocimiento durante la emergencia sanitaria (covid19)

\begin{tabular}{|c|c|c|c|}
\hline ÍTEMS & CANTIDAD & $\%$ & TOTAL \\
\hline CULTURAS & 25 & $20 \%$ & 25 \\
\hline INVESTIGAR & 52 & $43 \%$ & 52 \\
\hline POEMAS & 2 & $2 \%$ & 2 \\
\hline PASAR EL TIEMPO & 43 & $35 \%$ & 43 \\
\hline
\end{tabular}

Elaborado por: Autoras de la investigación (2021).

Fuente: estudiantes de tercero de bachillerato del Colegio "Pedro Zambrano Barcia"

\section{Gráfico 4. Lectura digital y el desarrollo de conocimiento}

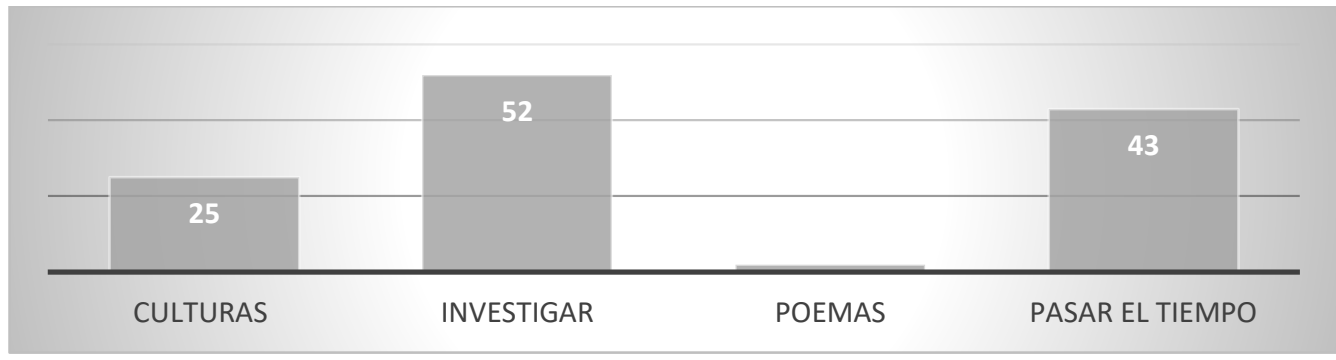

Elaborado por: Autoras de la investigación (2021).

Fuente: estudiantes de tercero de bachillerato del Colegio "Pedro Zambrano Barcia" 
Análisis en interpretación.

Se puede apreciar que el $43 \%$ del total de los estudiantes consideraron que la emergencia sanitaria motivó a los educandos a conocer otras formas de investigar, el 35\% mencionaron que es otra forma de pasar el tiempo y el $20 \%$ manifestó que conocieron diferentes culturas. En este contexto, Paredes (2015), expresa que el acto de leer en un proceso significativo, donde tiende a desarrollar un espacio de lectura, en otras palabras, en la vida cotidiana, posee un conjunto de acciones de aprendizaje y luego su fijación, para convertirse en una necesidad. La persona que lee, se ve impulsado a indagar, es decir, siente necesario saber algo.

Tabla 5. Incidencia de la lectura

\begin{tabular}{lccc}
\multicolumn{1}{c}{ ÍTEMS } & CANTIDAD & $\%$ & TOTAL \\
\hline GUSTO POR LEER & 66 & $54 \%$ & 6 \\
\hline DISGUSTO POR LEER & 1 & $1 \%$ & 1 \\
\hline AUTOAPRENDIZAJE & 55 & $45 \%$ & 55 \\
\hline
\end{tabular}

Elaborado por: Autoras de la investigación (2021).

Fuente: estudiantes de tercero de bachillerato del Colegio "Pedro Zambrano Barcia"

\section{Gráfico 5. Incidencia de la lectura}

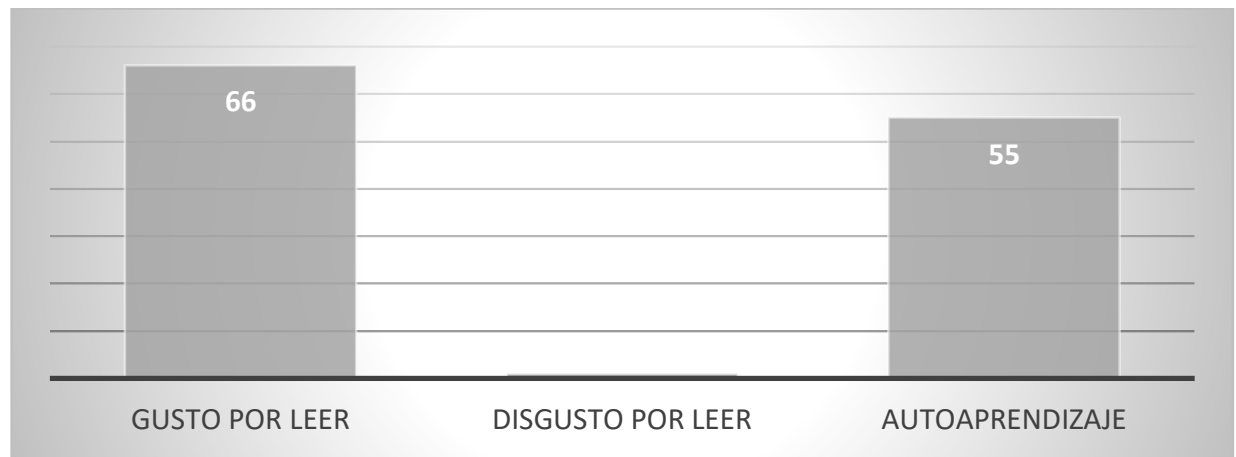

Elaborado por: Autoras de la investigación (2021).

Fuente: estudiantes de tercero de bachillerato del Colegio "Pedro Zambrano Barcia"

\section{Análisis e interpretación.}

Los resultados permiten mostrar que el $54 \%$ de los estudiantes respondieron que la lectura propició el gusto por ella y el $45 \%$ afirmó que conllevó al autoaprendizaje de cada uno de ellos. Ahora bien, Barba Chamorro Morillo Fuertes, \& Realpe (2016) mencionan que como habilidad comunicativa influye de manera directa en el proceso de desarrollo de la personalidad de los alumnos ya que además de modificar las particularidades de su pensamiento, imaginación, memoria y habilidades, también incide en sus sentimientos en sus emociones sus valores y comportamientos. Cuando se enseña a leer, se debe realizar partir de experiencias creativas, que conlleven a la creación de hábitos lectores adecuados en los alumnos. 


\section{Discusión de resultados}

De acuerdo a los resultados que se obtuvieron en la investigación, en la tabla número 1, se pudo verificar que el compartir con la familia se volvió una de las actividades más realizadas por los estudiantes que formaron parte de la investigación, es decir que la familia es un claro ejemplo de desarrollo de afectiva, y de significa para el desarrollo de actitudes y actividades que se dan dentro de este núcleo (Benítez, 2017). Que sin duda su función principal es la construcción desde su propio eje, Paladines \& Quinde (2010), reiteran que "la familia es una parte de la sociedad, en donde cada miembro evoluciona, se desenvuelve interactuando con factores internos (biológicos, emocionales, psicológicos) y externos (sociales), (p.17). A pesar que los modelos de familias han cambiado y se convirtieron en diferentes categorías, su desarrollo y su vínculo durante la pandemia ha sido una de las fuentes más específicas para la superación de los problemas presentados durante la pandemia; es decir los fenómenos sociales ayudan a interceder para mejorar su propia estructura (Gutiérrez, 2016).

En la tabla 2, se determinó con los estudiantes objeto de estudio que al leer mejora la comprensión lectora, pues sin duda, es uno de los grandes beneficios que tiene como proceso de construcción de significados, lo que demanda de un lector dialógico, que interactúe con la información del texto poniéndose en diálogo con el autor, formulando preguntas, hipótesis, haciendo inferencias, adoptando un planteamiento crítico (Gutiérrez, 2016). Hay que considerar a la lectura esencial en el desarrollo y formación para los jóvenes para poder enfrentar a los desafíos en la era del conocimiento, en los niveles académicos es necesario para el óptimo aprovechamiento de los alumnos, de la misma manera para el mejoramiento de su desempeño intelectual y cognitivo. (Flores, 2016).

Con los resultados de la tabla 3, se constató que los estudiantes optaron por leer mediante libros digitales, puesto que la pandemia también obligó a los lectores a conseguir este tipo de textos. Nunca han concitado tanta atención éstos y la lectura como ahora, en que los cambios tecnológicos y la posibilidad de una migración similar a la producida en otros géneros, como las obras de referencias y las revistas científicas, preferentemente, han movilizado a una gran parte de los actores implicados en la detracción o defensa de las transformaciones presentes o venideras (Córdon, 2018).

Por otro lado, en la tabla número 4, se verificó que los educandos tienen más interés por investigar como producto de su hábito lector, esto sucedió a raíz de la emergencia sanitaria. El ser humano busca estar informado constantemente sobre el mundo y los avances tecnológicos, y para que tenga éxito, hay que fomentar la lectura, para dar seguridad, permitir prepararse y tener conocimiento acerca de lo que acontece en su localidad o fuera de ella. (Figueroa \& Zambrano, 2015)

Con la tabla número 5, se demostró la lectura despertó en los estudiante el gusto hacia ella, pues, se convirtió en una sana costumbre y la imaginación que ésta despierta en el lector, es fructífero. Guerrero, Valledor \& Ponce de León (2018), mencionan que el hábito desarrolla en la persona la metacognición de acuerdo a lo que realiza cotidianamente. Osorio (2019) argumenta que el hábito lector, va más allá de su propio aprendizaje 
técnico, debido a que supone el paso de leer esencialmente pasivo a hacerlo vivo y activo, que profundiza en el texto, intercambia experiencias e inquietudes.

\section{Conclusiones.}

- La pandemia ha dejado secuelas negativas en diferentes aspectos, producto de ello, la sociedad se sometió a tomar medidas extremas con el fin de protegerse y una de ellas fue no salir de casa. Sin embargo, el confinamiento ha sido una oportunidad de compartir mucho más en familia como quedó demostrado en esta investigación donde los estudiantes supieron expresar las actividades que realizaban en cuarentena.

- Por otro lado, el acto de leer se ha convertido en estos tiempos en un pasatiempo favorito para los jóvenes especialmente, pues, es necesario recordar que la lectura enriquece de conocimiento, pensamientos y habilidades a quien le guste leer. Con las clases virtuales, se ha fomentado mucho más la lectura, resultando que los estudiantes la consideran un hábito y se interesan en seguir leyendo por su propia cuenta.

- El impacto de los hábitos lectores en los estudiantes fue altamente positivo, puesto que, durante la pandemia del COVID-19, tuvieron más tiempo libre para leer y algunos afianzaron su gusto por la lectura, otros lo desarrollaron y una gran parte potenciaron el placer por leer, por lo tanto, tuvieron un alto nivel de autoaprendizaje.

\section{Referencias bibliográficas.}

Aguirre, D., Zhindon, L., \& Pomaquero, J. (2020). COVID-19 y la Educación Virtual Ecuatoriana. Investigación Académica, 1(2), 53-63. http://investigacionacademica.com/index.php/revista/article/view/24

Alvarado, G. (2016). La importancia de la lectura infantil mediante actividades lúdicas para el desarrollo cognitivo en niños y niñas de 5 años del sur de Guayaquil, [Tesis, Universidad Laica Vicente Rocafuerte de Guayaquil]. http://repositorio.ulvr.edu.ec/handle/44000/1245

Alonso, M. \& Frederico, A. (2020). El rol de las bibliotecas en tiempos de COVID-19: reflexiones y propuestas. Desde el Sur. 12(1), 241-262. http://www.scielo.org.pe/scielo.php?pid=S2415$09592020000100241 \&$ script=sci_arttext\&tlng=pt

Barba, M., Chamorro, F., Morillo, J., Fuertes, J., \& Realpe, J. (2016). La promoción de la lectura desde la docencia. Una necesidad en Universidad Regional Autónoma de Los Andes en Tulcán, Ecuador. Didáctica y Educación, 7(2), 205-220. https://dialnet.unirioja.es/servlet/articulo?codigo $=6644654$

Blum, S. (2020). Matrimonio y cuarentena ¿combinación perfecta? https://www.youtube.com/watch?v=MJTgiQGcwtM 
Camacho, G. (2016). El hábito de la lectura en la comprensión lectora de los estudiantes de la Unidad Educativa Quisapcincha, cantón Ambato, [Tesis, Universidad Técnica de Ambato]. https://repositorio.uta.edu.ec/handle/123456789/23200

Caride, J. P. (2015). Leer el mundo hoy o cuando la lectura se convierte en diálogo. Ocnos: Revista De Estudios Sobre Lectura, (14), 65-80. https://revista.uclm.es/index.php/ocnos/article/view/ocnos_2015.14.05

CEPAL. (2020). La educación en tiempos de la pandemia de Covid-19, https://repositorio.cepal.org/bitstream/handle/11362/45904/S2000510_es.pdf?se quence $=1 \&$ is Allowed $=\mathrm{y}$.

Córdon, J. (2018). Libros electrónicos y lectura digital: los escenarios del cambio. Palabra Clave. 7(2). http://sedici.unlp.edu.ar/handle/10915/66768

De Souza, T. (2016). Lectura, biblioteca e inclusión social: importancia de la promoción de la lectura en comunidades ribereñas en Amazonas, Brasil. Información, $\begin{array}{llll}\text { Cultura } & y & \text { Sociedad, } & \text { 93-106. }\end{array}$ http://revistascientificas.filo.uba.ar/index.php/ICS/article/view/2256

Del Valle, V. (2016). El acto de leer: una experiencia en Educación Primaria. Educere, 20(65), 91-98. https://www.redalyc.org/pdf/356/35646429010.pdf

Durango, Z. (2015). La lectura y sus tipos. Portal de las palabras, (1), 9-13. http://revistas.curn.edu.co/index.php/portaldelaspalabras/article/view/589/44

Estructura de Recerca Interdisciplinar ERI-Lectura. (2020). Hábitos lectores durante el confinamiento por la covid-19 [Informe ejecutivo]. RODERIC (Universitat de València). Disponible en: http://roderic.uv.es/

Figueroa, W., \& Zambrano, P. (2015). Análisis de la importancia de la lectura en la sociedad actual, https://www.gestiopolis.com/wpcontent/uploads/2016/10/analisis-importancia-lectura-sociedad-actual.pdf

Flores, D. (2016). La importancia y el impacto de la lectura, la escritura y el pensamiento crítico en la educación superior. Zona Próxima, (24), 128-135. http://www.scielo.org.co/scielo.php?pid=S2145-

$94442016000100010 \&$ script $=$ sci_abstract\&tlng=en

Gagliardi, V. (2020). Desafíos educativos en tiempos de pandemia. Cuestión, 1-6. http://sedici.unlp.edu.ar/handle/10915/99685

Guardiola, E., Baños, J. (2021). La lectura durante la pandemia de covid-19. Revista de $\begin{array}{llll}\text { medicina } & y & \text { cine } & \text { 7-12. }\end{array}$ https://revistas.usal.es/index.php/medicina_y_cine/article/view/rmc202016e0712 
Guerrero, J., \& Ponce de León, R. (2018). La formación y desarrollo del hábito lector en Opuntia Brava, $8(3)$,

25-32. http://200.14.53.83/index.php/opuntiabrava/article/view/259

Gutiérrez, R, Díaz, K, Román, R. (2016). El concepto de familia en México: una revisión desde la mirada antropológica y demográfica. Ciemcia, ergo-sum, 23 (3).219-230. El concepto de familia en México: una revisión desde la mirada antropológica y demográfica (redalyc.org)

Gutiérrez, R. (2016). La lectura dialógica como medio para la mejora de la comprensión lectora. Investigaciones sobre Lectura. (5), 52-58. https://www.redalyc.org/pdf/4462/446243923005.pdf

Jiménez, M., Fierro, B., \& García, A. (2016). La lectura y su promoción desde diferentes escenarios pedagógicos. Revista amauta, 14(28), 25-36. http://investigaciones.uniatlantico.edu.co/revistas/index.php/Amauta/article/view /1603Benítez, M. (2017). La familia desde lo tradicional a lo discutible. Novedades en población, 26. 58-68. rnp050217.pdf (sld.cu)

Hilario, G. (2016). La animación a la lectura en la formación del hábito lector en estudiantes de Cuarto y Quinto grados de Educación Secundaria de la I.E.P. "Bertolt Brecht" S.J.L., 2014, [Tesis de maestría, Universidad Católica Sedes Sapientiae]. http://repositorio.ucss.edu.pe/handle/UCSS/189

Paladines, M, Quinde, M. (2010). Disfuncionalidad familiar en niñas y su incidencia en el rendimiento escolar. (Tesis de pregrado, Universidad de Cuenca). Cuenca, Ecuador. TESIS (ucuenca.edu.ec)

Torres, J. (2020). Propuestas para el mejoramiento de la educación universitaria virtual después del brote del COVID-19. Revista UVserva, año 5, número 9. http://uvserva.uv.mx/index.php/Uvserva/article/view/2700

Velázquez, L. (2020). La COVID-19: reto para la ciencia mundial. Anales De La Academia De Ciencias De Cuba, 10(2), e763. http://revistaccuba.sld.cu/index.php/revacc/article/view/763/792

Venegas, G. (2019). La Motivación (según la Teoría de Maslow) y el hábito lector en los estudiantes del segundo grado de secundaria en la I.E. "Sagrado Corazón de Jesús" del Cusco, [Tesis, Universidad Nacional Pedro Ruiz Gallo]. http://repositorio.unprg.edu.pe/handle/UNPRG/8162

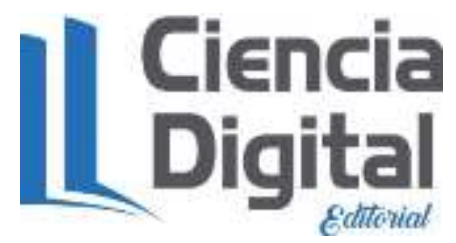




\section{PARA CITAR EL ARTÍCULO INDEXADO.}

Macías Mendoza, L. G., \& Viviana Navarro Cedeño, V. (2021). Impacto de los hábitos lectores en los estudiantes de bachillerato en época del covid-19. 2020. ConcienciaDigital, 4(4), 70-84. https://doi.org/10.33262/concienciadigital.v4i4.1894

\section{LCiencia}

El artículo que se publica es de exclusiva responsabilidad de los autores y no necesariamente reflejan el pensamiento de la Revista Conciencia Digital.

El artículo queda en propiedad de la revista y, por tanto, su publicación parcial y/o total en otro medio tiene que ser autorizado por el director de la Revista Conciencia Digital.

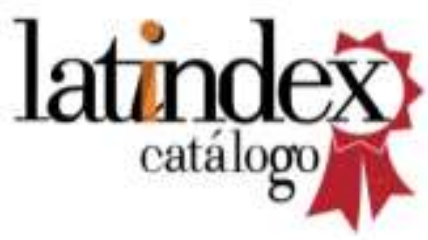

